\title{
Multilinguales
}

\section{La dimension interculturelle dans l'enseignement/ apprentissage du français en Algérie entre représentations et connaissances culturelles}

The intercultural dimension in teaching/learning french in Algeria between representations and cultural knowledge

\section{Nabila Hamidou}

\section{(2) OpenEdition Journals}

Édition électronique

URL : http://journals.openedition.org/multilinguales/1631

DOI : $10.4000 /$ multilinguales. 1631

ISSN : 2335-1853

\section{Éditeur}

Université Abderrahmane Mira - Bejaia

\section{Édition imprimée}

Date de publication : 1 juin 2014

Pagination : 125-138

ISSN : 2335-1535

Référence électronique

Nabila Hamidou, "La dimension interculturelle dans l'enseignement/apprentissage du français en Algérie entre représentations et connaissances culturelles », Multilinguales [En ligne], 3 | 2014, mis en ligne le 03 juin 2014, consulté le 17 septembre 2019. URL : http://journals.openedition.org/ multilinguales/1631; DOI : 10.4000/multilinguales.1631

Ce document a été généré automatiquement le 17 septembre 2019.

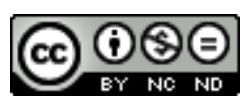

Multilinguales est mise à disposition selon les termes de la Licence Creative Commons Attribution Pas d'Utilisation Commerciale - Pas de Modification 4.0 International 


\section{La dimension interculturelle dans l'enseignement/apprentissage du français en Algérie entre représentations et connaissances culturelles}

The intercultural dimension in teaching/learning french in Algeria between representations and cultural knowledge

\section{Nabila Hamidou}

Dans une perspective interculturelle, l'enseignement d'une langue étrangère exige la prise en considération des éléments historiques, géographiques et ethniques. Il n'est plus possible que les élèves (apprenants) réfléchissent à leur "vivre ensemble» de manière "mono-culturelle». On peut supposer incontournable qu'ils s'ouvrent aux autres, à l'altérité, au dialogue; qu'ils s'intéressent aux métissages culturels et qu'ils comprennent que le monde dans lequel ils vivent est un monde à partager.

Parler de dimension culturelle dans l'enseignement d'une langue en général et d'une langue étrangère en particulier est un fait tout à fait conséquent puisque cette dimension est derrière toute pratique langagière. La relation langue-culture est si étroite qu'elle constitue les deux faces d'une même pièce, ou mieux, d'une même page.

L'enseignement de la culture est sous-jacent à la pratique scolaire quotidienne de la langue cible.

4 Nous convenons donc avec Geneviève Zarate (1995) qu'on ne peut pas apprendre une langue en en évacuant la culture. Martine Abdallah-Preitcelle (2001) est du même avis. Elle s'interroge sur le fait que l'on puisse enseigner une langue étrangère sans l'ancrer dans sa culture. Pour elle, la dimension interculturelle de l'enseignement s'impose d'elle-même parce que dès que l'Autre est convoqué, comme ici à travers sa langue, 
nous sommes d'emblée dans la diversité culturelle. Elle préconise donc de mettre en œuvre des démarches pédagogiques pour en favoriser la prise de conscience.

Selon elle (2004), en effet, «le discours interculturel induit un questionnement autant sur les autres cultures, sur autrui, que sur sa propre culture. C'est ce processus en miroir qui fonde la problématique interculturelle» (p. 28).

6 La représentation que nous avons de celui qui parle la langue que nous avons à apprendre influence considérablement l'apprentissage. Il convient d'en prendre conscience et de développer des pistes pédagogiques dans ce sens.

7 Nous allons, dans le cadre de cet article, traiter de la dimension interculturelle dans l'enseignement de la langue française en Algérie, à travers une enquête faite auprès d'enseignants et d'apprenants de la région Ouest de l'Algérie.

Pour des résultats fiables, nous avons évité dans un premier temps toute inférence du type: "il se pourrait que tel enseignant ait dit cela parce que... ", nous limitant à l'explicite observable. Il ne s'agit pas de spéculer sur les non-dits mais d'analyser ce qui est clairement exprimé. L'interprétation ne surviendra que dans un second temps.

Compte tenu de quelques difficultés d'ordre linguistique de certains apprenants, nous leur avons permis de répondre dans leur langue maternelle. Pour ceux qui ont répondu en langue française, nous pensons qu'il faudrait s'attacher davantage à la signification de la réponse et non à ses signifiants. Autrement dit, nous avons mis de côté l'aspect formel des réponses telles le lexique, l'orthographe et la syntaxe.

Les résultats de l'enquête que nous avons menée révèlent que ce qui est surtout ciblé en classe de langue c'est la maitrise de la langue dans sa seule dimension linguistique. La dimension socioculturelle quant à elle est mise à l'écart par beaucoup d'enseignants et n'est abordée qu'accidentellement. En effet, $68 \%$ des élèves interrogés affirment qu'ils travaillent en classe de langue beaucoup plus sur la langue que sur le pays.

11 Concernant la conception de l'apprentissage culturel de la langue française, les enseignants sont restés très évasifs. D'aucuns disent qu'apprendre la culture véhiculée par la langue n'est ni nécessaire ni obligatoire pour la maîtrise de cette langue. D'autres se défendent en expliquant que les élèves ne s'y intéressent pas car ne posent aucune question quant à cette dimension.

12 Les résultats de l'enquête faite auprès des élèves montrent pourtant le contraire. En effet, ce qui ressort de l'analyse des réponses données par les apprenants, c'est le besoin de connaitre les français dans leur dimension anthropologique : avoir une idée sur leurs coutumes, leurs traditions, leur culture, leur façon de penser. Ils veulent aussi jeter un regard sur la politique et les lois françaises, avoir une idée sur l'histoire de cette nation, connaître sa géographie et ses monuments historiques. Nous avons été très surprise par les réponses de beaucoup d'apprenants.

En effet, leurs connaissances culturelles concernant ce pays qu'est la France restent très larges; ils connaissent les principales villes de France; ils citent dans leurs réponses tous les plus beaux monuments de Paris. La Tour Eiffel est en première position avant la capitale qui l'abrite, en deuxième position. Viennent ensuite les Champs Elysées en troisième position, puis d'autres villes.

14 Concernant leurs connaissances de la culture française, neuf personnalités célèbres ont été citées. Sont évoqués, dans l'ordre décroissant, Victor Hugo, Jacques Chirac, Zidane, Nicolas Sarkozy, Smaine, Napoléon, Molière, Jules Verne et Rousseau. 
de se représenter l'Autre à travers une image valorisante ou dévalorisante. Dans le cas qui nous intéresse, l'image de la France est celle d'un "pays de Cocagne ». Quant au Français, il apparaît dans l'ordre des réponses données, comme quelqu'un de raciste, mais instruit, cultivé, travailleur, sérieux, moderne, poli.

21 Autant de qualifiants qui nous installent toujours dans cette image valorisante de l'Autre, très souvent opposée à une image dégradante du moi. Or cette image comme toute image stéréotypée est très réductrice, et nous pensons que le premier souci de tout enseignant de langue étrangère est de faire prendre conscience à ses élèves de la relativité de leurs points de vue concernant les autres cultures.

A la question de savoir, de la composante socioculturelle et de la composante sociolinguistique, qu'elle est celle qui est inhérente aux objectifs visés, $38 \%$ des enseignants interrogés mettent l'accent sur la composante sociolinguistique, qualifiée de composante-clé. Cette composante est assimilée à la dimension cognitive (savoirs et savoir-faire) permettant d'anticiper et de gérer une diversité de situations auxquelles les élèves sont (et seront) confrontés, dans différents contextes et différentes situations où ils doivent réagir (répondre) le plus adéquatement possible, non seulement par l'utilisation de structures langagières adaptées, mais également par des comportements et des stratégies d'ajustement appropriés ${ }^{1}$.

Pour 40 \% d'entre eux, la langue ne peut être apprise de manière technique, c'est-à-dire sans les soubassements culturels que véhicule cette même langue. Sans le non-dit, la langue n'est qu'un corps sans âme. Ils affirment que la communication, met en jeux non seulement des dimensions verbales mais également d'autres, non-verbales, explicites et implicites, visibles et invisibles. Par ailleurs peut-on dissocier les deux dimensions?

«La compétence sociolinguistique renvoie aux paramètres socioculturels de l'utilisation de la langue. Sensible aux normes sociales (règles d'adresse et de 
politesse, régulation des rapports entre générations, sexes, statuts, groupes sociaux, codification par le langage de nombre de rituels fondamentaux dans le fonctionnement d'une communauté), la composante sociolinguistique affecte fortement toute communication langagière entre représentants de cultures différentes, même si c'est souvent à l'insu des participants eux-mêmes. » (p. 18) culture de l'Autre.

NOUS POUVONS DIRE, EN EFFET, QUE, DANS LE CONTEXTE DE COMMUNICATION, LA COMPETENCE LINGUISTIQUE ASSOCIE LES DEUX COMPOSANTES, SOCIOCULTURELLE ET SOCIOLINGUISTIQUE, QUI DEVIENNENT AINSI INDISSOCIABLES.

Mais, dans les programmes de 1re année secondaire, les compétences de communication visées sont essentiellement sociolinguistiques. Il s'agit de faire acquérir à l'apprenant des savoirs linguistiques et des savoir-faire discursifs.

Cependant, puisque " la conception communicative de la langue doit être (re) contextualisée dans ses usages sociaux» (Gohard - Radenkovic, 1999: 185), l'ensemble de ces compétences nécessite la maîtrise d'autres compétences comme les compétences socioculturelles qui sont à la base des compétences comportementales. Ainsi donner des informations, exprimer un point de vue ou bien raconter des événements fictifs ou en relation avec le vécu, comme cela est inscrit dans le programme de 1re AS, suggère que l'apprenant soit déjà familiarisé avec les règles de conversation dans la langue étudiée.

8 De la même façon, les textes officiels algériens, comme le Référentiel Général des Programmes (2006) du ministère de l'éducation nationale, insistent sur la nécessité d'appréhender l'Autre sans parti pris: «Les programmes éducatifs doivent inscrire, au nombre de leurs objectifs, l'information objective sur les cultures, les civilisations, l'évolution des métiers, des professions et des marchés de l'emploi (Référentiel des programmes » (p. 11).

9 Le discours de la plupart des enseignants, quant à cette question, rejoint indubitablement le discours politique dans sa conception de l'enseignement / apprentissage d'une langue. Ils s'accordent sur le fait que l'essence même de l'apprentissage d'une langue étrangère est tout son contenu culturel, toute la vision du monde qu'elle véhicule.

Dans cette optique, l'enseignant doit se considérer, selon l'expression de Zarate et Byram (1997: 09), comme «un intermédiaire culturel» entre sa propre culture et la

Cependant, la réalité de la classe est tout autre. Ainsi pour la question de savoir si pour l'apprenant, il était nécessaire de connaître la culture sous-jacente à la langue étudiée, $80 \%$ des enseignants répondent par l'affirmative ; les autres l'occultent complètement pendant leurs cours et ne se préoccupent que de la seule dimension linguistique.

Pourtant, les pédagogues, les textes officiels, comme nous l'avons souligné, recommandent d'enseigner la langue étrangère en l'insérant dans son contexte culturel. Cette façon d'aller à la rencontre de l'Autre devrait être privilégiée dans l'enseignement des langues étrangères parce qu'elle favorise à la fois la maîtrise technique en même temps qu'une meilleure connaissance de la culture des pays concernés. Ce bénéfice est d'autant plus appréciable qu'il prépare l'apprenant à la mobilité internationale, clé de voûte du nouveau dispositif de l'enseignement universitaire en Algérie ${ }^{2}$.

$3389 \%$ des enseignants sondés estiment que l'objectif principal de l'enseignement / apprentissage d'une langue est de développer la disponibilité et la volonté de communiquer avec l'Autre. Ils affirment qu'apprendre une langue étrangère, c'était 
construire un pont qui relie deux interlocuteurs différents afin de comprendre l'autre et de l'accepter avec sa différence; ou encore que c'était un pas vers l'ouverture et la connaissance de l'Autre; d'autres vont plus loin en répondant que le salut des nations serait aujourd'hui dans l'interculturalité car la planète était devenue un petit village.

Quelles que soient les réponses données par les enseignants, tous s'accordent à dire que cet état de fait est dû à la mondialisation qui l'impose et que grâce aux nouvelles technologies, les hommes de toutes les nations peuvent communiquer entre eux et s'ouvrir sur le monde.

"Communiquer, c'est entrer en relation avec l'Autre, l'ailleurs, le différent, c'est instaurer un mouvement à double polarité, un dialogue au sens technique du terme», selon Martine Abdellah-Pretceille et Louis Porcher (2001: 5). Notons bien l'expression «entrer en relation » qui suppose une relation d'échange, de contact et de correspondance.

Zarate (1995), définit la classe de langue dans le même sens : « un des lieux où la culture du pays de l'élève et la culture étrangère enseignée entrent en relation » (p.11).

Ainsi, l'enseignement/ apprentissage d'une langue étrangère ne peut se concevoir sans un rapport à l'Autre et la loi d'orientation sur l'éducation nationale du 23 janvier 2008 du ministère algérien de l'éducation nationale, parue au journal officiel va dans le même sens.

En effet, dès le titre premier, "Les fondements de l'école algérienne ", "Les finalités de l'éducation », il est précisé que

«L'école algérienne a pour vocation de former un citoyen doté de repères nationaux incontestables, profondément attaché aux valeurs du peuple algérien, capable de comprendre le monde qui l'entoure, de s'y adapter et d'agir sur lui et en mesure de s'ouvrir sur la civilisation universelle. » (Chapitre 1, art. 2)

Cette finalité d'ouverture de l'école sur les autres civilisations revient explicitement, dans le texte de cette loi, à l'enseignement des langues étrangères :

«Dans le cadre de sa mission fixée à l'article 44 ci-dessus, l'enseignement fondamental vise, notamment, à permettre la maîtrise d'au moins deux langues étrangères en tant qu'ouverture sur le monde et moyen d'accès à la documentation et aux échanges avec les cultures et les civilisations étrangères. » (Titre III, Chapitre III, art.45)

40 Apprendre la langue de l'Autre aide l'apprenant à communiquer avec lui en ayant une meilleure compréhension de sa culture et de son mode de penser.

41 Nous avons voulu savoir si les enseignants faisaient une quelconque différence entre enseignement de la culture et formation à une compétence interculturelle. Sous la rubrique "néant ", nous avons regroupé les enseignants qui ont affirmé ne pas savoir et ceux qui n'y ont pas répondu du tout. Ils représentent une proportion de $39 \%$.

Nous considérons les non-réponses comme une inaptitude de la part de ces enseignants à réagir à cette question par méconnaissance du domaine. $36 \%$ des enseignants interrogés ont donné des réponses erronées. Entre les réponses fausses et les nonréponses, nous constatons que $75 \%$ des enseignants ignorent la différence qui peut exister entre enseignement de la culture et formation à la compétence interculturelle.

Les réponses données par les $25 \%$ restants sont très variées, mais elles convergent toutes vers un point commun : former l'apprenant à la compétence interculturelle, c'est lui apprendre à connaître et à reconnaître l'Autre, à s'ouvrir sur les idées et les 
croyances des autres, à échanger avec l'Autre et construire ses relations avec les autres populations du monde.

Quant à l'enseignement de la culture, ces enseignants le ramènent tous à l'enseignement des normes et des valeurs d'une société.

Il ne s'agit pas de transmettre uniquement des connaissances culturelles mais d'aller au-delà, dans une optique interculturelle où la compétence de communication " reposera sur la capacité des interlocuteurs à repérer le culturel dans les échanges langagiers " (ibidem.). Il est ainsi indispensable de dépasser le civilisationnel dans l'apprentissage d'une langue pour intégrer toute une vision du monde révélatrice des différences et des similitudes entre deux ou plusieurs cultures. Ainsi,

«Dans une approche interculturelle, un objectif essentiel de l'enseignement des langues est de favoriser le développement harmonieux de la personnalité de l'apprenant et de son identité en réponse à l'expérience enrichissante de l'altérité en matière de langue et de culture. » (Conseil de L'Europe, 2001 : 09)

A la question de savoir quelles activités mettre en place en classe en vue d'asseoir une compétence interculturelle et quels supports choisir, $90 \%$ des réponses données par les enseignants mentionnent uniquement les supports sans spécifier les tâches. Ils assimilent le support à la tâche, mais n'en donnent aucune description.

Les $10 \%$ restants citent des activités proposées aux apprenants de manière générale et très vague. Quant aux supports utilisés, les textes littéraires et scientifiques restent les plus prisés.

Partant du postulat selon lequel l'apprentissage interculturel repose sur la communication, le vécu commun et l'intérêt de connaître l'Autre, nous pensons que pour faire prendre conscience aux apprenants des différences et pour développer la capacité de communiquer efficacement avec ceux qui sont différents, l'adoption d'un enseignement de type transversal et l'introduction de situations réelles devraient être prises en considération. Pour cela, des activités qui impliqueraient l'apprenant devraient être développées. Il devient nécessaire de réfléchir à des situations dans lesquelles l'apprenant puisse accomplir des tâches à finalité collective.

Dans une vision interculturelle, certains enseignants interrogés parlent de partir effectivement d'une situation réelle de la culture source et d'aller vers la culture cible en utilisant la même situation (exemple : le temps et l'espace). D'autres estiment devoir utiliser le cinéma, le théâtre, la chanson, la littérature et la poésie, pour installer cette compétence chez l'apprenant. D'autres encore préfèrent recourir à des textes de différents auteurs appartenant à des cultures différentes avec des thèmes variés et motivants pour l'apprenant.

D'après Abdellah-Pretceille M. (2004) :

«L'inter-relation de la langue et de la culture, depuis longtemps reconnue par les ethnologues et les anthropologues, est considérée désormais comme un point d'ancrage de l'enseignement de toute langue vivante. Il ne s'agit plus de juxtaposition des apprentissages, mais de complémentarité.» (p. 170)

51 Cette inter-relation peut se faire à travers l'étude de l'histoire du pays, de sa géographie, de sa société et de sa littérature, etc. Mais il faudrait prendre conscience aussi, que "pour appréhender une culture, [il nous faut] tenir compte des transformations politiques et sociales" (ibidem, p.171). L'internationalisation de la vie a fait que les sociétés entrent en contact et se transforment. C'est pourquoi J.-P. Warnier (2004) pense que « les langues et les cultures changent, car elles sont immergées dans les turbulences 
de l'histoire. Afin d'assurer leur fonction d'orientation, elles doivent intégrer le changement » ( $\mathrm{p}$. 13). connaître la culture véhiculée par cette langue. Cette connaissance est nécessaire à l'apprentissage de la langue, comme la connaissance de cette dernière est nécessaire à l'accès à la culture.

57 C'est grâce à ce lien interculturel que les apprenants réalisent l'altérité comme une ouverture de soi et vers autrui. Convenons avec Michel Serres que «tout apprentissage suppose une inclusion, un accueil, et je décris simplement l'idéal de l'éducation comme l'ouverture à toutes les altérités possibles $»^{4}$. travers une éducation dont l'objectif est cette «inclusion de l'Autre» dont parle M. Serres.

59 L'apprentissage des langues permet de «voir» les différences dans les formes d'expressions du monde. Ce processus est multifonctionnel dans le sens où il permet non seulement de découvrir une langue et sa culture mais également d'intégrer les différences dans sa propre culture. sienne propre et qui est différente de celles des autres sociétés. 
64 L'individu s'émancipe et son épanouissement se fait grâce à cette altérité qui tend à percevoir l'Autre comme l'autre de soi. Ignorer la culture de l'Autre ou la rejeter par convictions religieuses ou idéologiques ne peut qu'engendrer des formes extrêmes, telles que le racisme et la violence. Aujourd'hui, la mondialisation touche de manière significative la vision traditionnelle des échanges entre les peuples, et, entre autres, celles liées aux identités culturelles et aux modes de penser et d'agir.

L'introduction du plurilinguisme est devenue l'une des tendances mondiales de l'éducation. C'est pour cette raison, qu'en matière d'enseignement des langues étrangères, qu'il est devenu impératif d'appliquer le principe d'ouverture sur les cultures du monde rappelée à plusieurs endroits de la loi d'orientation évoquée cidessus.

La mondialisation a permis à l'enseignement/apprentissage des langues étrangères de se redéployer. Il doit permettre aux élèves algériens d'accéder directement aux connaissances universelles, et de s'ouvrir à d'autres cultures.

Il est ainsi clair que la portée culturelle et civilisationnelle d'une langue ne peut être ignorée, et doit être saisie à travers son apprentissage.

\section{BIBLIOGRAPHIE}

ABDELLAH PRETCEILLE Martine, L'Education interculturelle, Paris, PUF, 1999 (1ère édition), 2004 (2ème édition).

ABDELLAH PRETCEILLE Martine \& PORCHER Louis, Education et communication interculturelle, Paris, PUF, 1996 (1ère édition), 2001 (2ème édition). BEACCO Jean Claude, Les dimensions culturelles des enseignements de langue, Paris, Hachette, 2000.

CONSEIL DE L'EUROPE, Cadre Européen Commun de Référence pour les langues : apprendre, enseigner, évaluer, Paris, Didier, 2001.

Disponible sur HYPERLINK : http://www.coe.int/t/dg4/linguistic/source/frameword_fr.pdf WARNIER Jean-Pierre, La mondialisation de la culture, Paris, La Découverte, 2003, (réédition 2004).

ZARATE Geneviève, Représentations de l'étranger et didactique des langues, Paris, Didier, 2004.

REFERENTIEL GENERAL DES PROGRAMMES, document émanant du Ministère algérien de l'éducation nationale, 2006.

\section{WEBOGRAPHIE}

- Zarate.G, Byram.M, Neuner.G, « Définitions, objectifs et évaluation de la compétence socioculturelle », dans M. Byram, G. Zarate et G. Neuner (Dirs), La compétence socioculturelle dans l'apprentissage et l'enseignement des langues, Strasbourg, Edition du conseil de l'Europe, 1997, pp. 9-41. Disponible sur : www.coe.int/t/dg4/linguistic/Source/SourcePublications/ CompetenceSocioculturelle_FR.doc 
- Conférence du CAP, 11-13 octobre 2002. «Favoriser la diversité culturelle et le développement: stratégies locales, nationales et mondiales ».Consultable sur le site : www. incd.net/ docs/Cape TownDeclarationF.htm

- Loi n 08-04 du 15 Moharram 1429 correspondant au 23 janvier 2008 portant Loi d'Orientation sur l'Education nationale, Journal Officiel $n^{\circ} 4$ du 23 janvier 2008. Consulté sur le site :

$<$ www.joradp.dz/FTP/jo-francais/2008/F2008004.pdf<

\section{NOTES}

1. Selon Evelyne Rosen, dans son article intitulé «La mort annoncée des "quatre compétences" pour une prise en compte du répertoire communicatif des apprenants en classe de FLE », " $L a$ compétence sociolinguistique renvoie à la fois aux règles socioculturelles et aux règles discursives, c'est-àdire à la capacité de produire et de comprendre des énoncés appropriés à des situations sociales spécifiques et conformes aux codes sociaux de ces situations ", in Glottopol, $\mathrm{n}^{\circ}$ 6, juillet 2005, pp. 120-133. Consulté sur le site : glottopol.univ-rouen.fr/telecharger/numero_6/gpl6_07rosen.pdf

2. Il s'agit du dispositif LMD (Licence - Master - Doctorat) mis en place à l'université en 2004.

3. Conférence du CAP, 11-13 octobre 2002. www.incd.net/docs/CapeTownDeclarationF.htm. Trois jours de discussions ont regroupé 186 délégués de 37 pays autour du thème « Favoriser la diversité culturelle et le développement : stratégies locales, nationales et mondiales "

4. Interview de Michel Serres, en 1993, dans l'Hebdo, à l'occasion de la parution de son livre, « La Légende des Anges». Consultable sur le site: <wwwedu.ge.ch/CPTIC/publications/formntic/ vert08.html>

\section{RÉSUMÉS}

Enseigner une langue est pensé dans son essence comme une activité culturelle. Enseigner une ou plusieurs langues, c'est éduquer à une diversité linguistique, et par là culturelle. De plus, l'éducation à la diversité linguistique et culturelle est une éducation au dialogue et à la tolérance, et favorise la communication interculturelle L'enseignement de la culture est nécessaire à l'apprentissage d'une langue, comme la connaissance de cette dernière est nécessaire à l'accès à la culture. C'est grâce à ce lien interculturel que les apprenants réalisent l'altérité comme une ouverture sur soi et sur autrui.

Teaching language in essence is conceived a cultural activity. To teach one or several languages is to educate for linguistic diversity and hence cultural diversity. Furthermore, education for linguistic and cultural diversity is an education for dialogue and tolerance. It also enhances cross-cultural communication. Teaching culture is necessary for language learning, as knowing language is indispensable for accessing culture. It is thanks to this cross-cultural link that learners understand otherness as openness to oneself and others. 
INDEX

Mots-clés : enseignement, apprentissage, langue française, représentations, interculturel

Keywords : teaching, learning, french language, representations, cross-cultural

\section{AUTEUR}

\section{NABILA HAMIDOU}

Université d'Oran

HAMIDOU Nabila est maître de conférences en didactique des langues, à l'université d'Oran (Algérie). Elle est membre du laboratoire de recherche « langue française au Maghreb - pratiques de suivi et évaluation de compétences en francophonie » (LAFRAMA).

Elle a publié de nombreux articles surtout dans le domaine de la didactique des langues dont : - « Du programme de français... à l'enseignement de la langue en 1ère A.S », in Resolang, $n^{\circ} 1$, Université d'Oran/Université de Lyon, 2008, pp. 115-122.

- "L'altérité comme valeur sûre de l'enrichissement individuel », in Résolang, n 3, Université d'Oran/Université de Lyon, 2009, pp. 69-77.

- « Le manuel dans l'institution scolaire. Approche pédagogique ", in Resolang, n 4, Université d'Oran/Université de Lyon, 2009, pp. 97-104.

- « Le manuel de 1èreAs comme espace d'une représentation de soi et de l'autre », in revue Insaniyat, $\mathrm{n}^{\circ} 43$, juillet 2009.

- «Pour un enseignement interculturel de la langue française en Algérie dans un contexte de mondialisation ", in Passerelles, n 6, Anwar Eaârifa, 2013. 